\title{
Using Clinical Utilisation Review and quality improvement methodology improves patient flow in the acute hospital setting
}

\author{
Authors: Emma Vardy, Debbie Meehan, James Barham, Imogen Lyons and Hilary Richardson
}

\section{Introduction}

Clinical Utilisation Review (CUR) is an evidence-based tool that identifies if a patient is cared for at the correct level of acuity at the time of assessment. The tool identifies internal delays which are occasioned by delays inherent in the acute setting; it identifies external delays which are outside the control of the acute hospital. CUR identifies delays to discharge and individual patient journeys. It identifies themes and areas where resource may be better placed to affect high-quality care. We present a project in which CUR data was used to improve patient flow on an acute geriatric medicine ward.

\section{Methods}

Ward $L 4$ is a 24 -bedded inpatient geriatric medicine unit. The nursing team input CUR data daily for patients selecting an appropriate reason delay code. Data was collated by the CUR project team and in October 2018 was presented to the ward multidisciplinary team showing the top 10 reasons for delay to discharge. A driver diagram was developed, focusing initially on three delay codes: fast track referral, best interest meetings and intermediate care (IMC) referrals. These delays were deemed within the remit of the multidisciplinary team to change.

The ward quality improvement (QI) team met regularly to review data, identify potential changes and plan Plan, Do, Study, Act (PDSA) cycles. Where relevant, the ward team engaged other teams to support. For example, process mapping of fast track referral with a member of the continuing healthcare team was used to identify issues resulting in delays and potential improvements. As the project progressed, the top reasons for delay shifted and additional themes were incorporated.

\section{Results and discussion}

Over a quarter, the project resulted in a $10 \%$ reduction in mean proportion of beds occupied by patients who were categorised as non-qualified (ie not requiring the level of care provided on the ward). Ward transfers increased from an average of 97 per month to 144 in January 2019. There was no compromise of friends and family feedback or concomitant increase in 28-day readmissions.

A reduction in delays from all three delay code categories was observed. This was estimated to save approximately 35 bed nights when compared with the previous quarter. The project resulted in a new streamlined process for referral to IMC across the trust with associated time and cost savings, particularly for the therapy team.

The project showed that delay in discharge is multifactorial and requires persistent focus of the multidisciplinary team to show improvement. The top 10 delays changed over the study period and new delay codes are being studied as part of an ongoing project.

\section{Conclusion}

We showed that CUR, combined with QI methodology, is an effective tool to help ensure patients receive the appropriate level of care in the correct environment at the correct time and can be used to improve inpatient flow. 